\title{
Promoting healthy musculoskeletal function by applying regular exercises education and physical activities in senior activity centers
}

\author{
Cindy Sin U Leong ${ }^{1 \mathrm{a}}$ \\ ${ }^{1}$ School of Health Sciences, Macao Polytechnic Institute, Rua de Luis Gonzaga Gomes, Macao
}

\begin{abstract}
There are varied types of exercises that are beneficial to promoting healthy musculoskeletal function among older adults. The purpose of this study was to investigate the factors contributing to the health of the adults by having regular exercise education and physical activities provided by the senior activity centers in Macao. 56 participants aged 65 and over from four senior activity centers were interviewed in the past two years. Three senior activity centers were located in Macao peninsula and one senior activity center was in Taipa, another island of Macao. Adults in the two senior activity centers provided with regular exercise education and physical activities were having better quality of life with more satisfaction in physical, psychosocial and mental development. The other two activity centers provided less or zero exercise program showed more chances of falls and health problems.
\end{abstract}

Keywords: Activity centers, regular exercises, healthy musculoskeletal function.

\section{Introduction}

Regular and healthy exercise is essential for maintaining musculoskeletal function and consequence independence reached among older adults. A few intrinsic risk factors of fall may include gait, chronic illness and side-effect of medication as well as balance impairment [1]. While extrinsic risk factor is mostly related to environment, therefore a good, comfortable and safe environment available for frail older adults is very important. Therefore, the living environment for outdoor activity and senior activity center for indoor activity is very supportive for older adults to keep on being social active and mobile. According to WHO [2], older adults' physical activity may include walking, dancing, swimming, household chores, and planned exercise as list a few. To the ideas of WHO, physical exercises have many benefits to not only for older adults' body development such as less fall incidence, better mobility balance, and psychosocial development [3]. Many studies have showed that regular exercise, for example as simple as walking, helps much in

\footnotetext{
${ }^{\text {a } C o r r e s p o n d i n g ~ a u t h o r: s u l e o n g @ i p m . e d u . m o ~}$
} 
body organ development which improves respiratory system, circulatory system and immune system[3-4]. Furthermore, the chronic illness has much control with medication treatment together, especially the diabetic mellitus sugar level in the blood is way better in control [5-6]. Referring back to the title of this paper, musculoskeletal function is much promoted by regular exercise. Improvement in musculoskeletal flexibility and strength among older adults assists to balance and reduce pain as their normal body function declines alongside aging. In consequence, the healthy physical status helps to postpone the occurrence of illness or prevent their chronic illness from getting worse. Moreover, the "good healthy body" let older adults feel the sense of well-being from psychosocial view [6]. As older adults often state that they feel satisfied with very simple daily life activities, such as they can "walk, stand, eat, and sleep", and do not need grown-up children to "care" them, which is a very good traditional thinking of Chinese older adults.

\subsection{Background of Macao}

Macao, a moderately-sized city of 645,000 residents who are predominantly Chinese (97\%), is approximately $35 \mathrm{~km}^{2}$ in size [7], and is located in the southern part of Guangdong Province. There are approximately 66,340 older adults over 65 years of age, which is around $9.8 \%$ of the Macao population. The average life expectancy of people in Macao is 87 years old for females and 81 for males. The average life expectancy of Macao residents is 84 years old. Therefore, the high life expectancy stimulates the author's interest to explore the senior activity centers' function, including the kind of exercise education and physical activities provided in the centres, as well as any difference or effects for the older adults who attend different senior activity centres with different mission of the centres. According to [8], quite many older adults in Macao were cared by non-family caregivers. Non-family caregivers would even bring the older adults out to park pushing wheelchair in the morning or late evening. Therefore, this phenomenon reminded the author those active life older adults might not require home maids' caring. There are many senior activity centers in Macao, and the author has the experience of knowing that many older adults would like to attend their nearby senior activity centers for leisure. The centers might have many different kinds of activities such as physcial education, interested class such as singing, dancing and drawing, just name a few. From the author's long-time observation, the older adults seemed more good-looking, healthier and more educated after attending the activities. Furthermore, the better life expectancy is having relationship with regular physical education taking in senior activity center. The author would also like to explore how older adults felt about their activity centers.

The aims of this study were to explore how the older adults perceive their physical education and how they felt about their activity centers' activities. The older adults' perception of their musculoskeletal function after having physical activities practicing would also be explored. As expected, the senior activity centers might provide more helpful and useful knowledge and skills to assist older adults to obtain better well-being.

\section{Method}

In this study, the data was collected from older adults who are 65 or above. The data was gathered through a qualitative approach. Many interviews were conducted among older adults who have participated in activities provided by senior activity centers. This qualitative study was approved by the Management Board of Research Committee at the Macao Polytechnic Institute. 


\subsection{Participants}

The study involved older adults from four senior activity centres. At the onset of the study, invitation letters were distributed to those senior activity centers for the explanation of this study. A total of four senior activity centers agreed to have interviews. Those older adults interested in the interviews signed the consent and understood their interviews were audiotaped anonymously or had private information recorded. Older adults aged 65 and above were invited for participation. There were 56 older adults willing to participate in the interview. Their age was from 65 to 94 years old. There were 43 females and 13 males recruited. All participants were married and had grown-up children, grandchildren and some participants even had great grandchildren. There were 10 females (a total of 23) aged 80 and over lived alone. All male participants lived with their wives.

Table 1. Participants from four senior activity centers

\begin{tabular}{|c|c|}
\hline Age group & Gender \\
\hline $65-69$ & $8 / 1$ \\
\hline $70-74$ & $5 / 4$ \\
\hline $75-79$ & $7 / 4$ \\
\hline $80-84$ & $14 / 1$ \\
\hline $85-89$ & $4 / 2$ \\
\hline $90-94$ & $5 / 1$ \\
\hline
\end{tabular}

$\mathrm{F}=$ female, $\mathrm{M}=$ male

\subsection{Interview}

The questions for the interviews (Table 1) were structured following literature reviews, past teaching observation and communication with the older adults. There are many reports mentioning that older adults having regular physical exercises and activities are less likely to have falls and more able to achieve physical balance. Accordingly, the questions explored what older adults had in their senior activity centers, how they felt and what they concerned the most during their physical exercises, such as pain, twist of their ankles and other concerns.

Table 2. Interview questions

1. Do your senior activity center provide regular exercise education and physical activity? Please describe.

2. How do you perceive your health? Please describe.

3. How do you perceive your exercise activity with your health status? Please describe.

4. Do you live with your family members? How do you make use of your time?

5. Does your apartment have elevators or you need to walk upstairs? If no elevators for your building, how many times a day did you walk? Do you feel tired? Please describe.

\subsection{Procedure}

To achieve a representative response, older adults from four different senior activity centers were selected. To be even more representative, the older adults were selected from centers located in Macao peninsular and Taipa (Macao is made up of three islands). Most Macao residents were living in these two big islands. The 56 interviewees included 43 females and 13 males. All participants welcomed the audio-taping of the interviews. Interviewing began two years ago. The interviews were limited to summer months (June to August regarding the researcher is an academic staff). At the beginning of each interview, the participant 
confirmed that the interview would be recorded. Participants were assured that their recorded material would be kept confidential. The interviews were conducted in Cantonese, after each interview the transcripts were analysed immediately to capture the researcher's thoughts and feelings. The participants were coded as numbers P01-P056.

\subsection{Transcription and data analysis}

All the interviews were transcribed into verbatim to ensure that the expression of the interviews was accurately reported. After transcription, the results were examined using thematic content analysis. The number of times that the same sub-concepts or concepts occurred in the interview was grouped into the same theme. For example, codes P1, P2, P3, $\mathrm{P} 4$, and so forth, were assigned to the concepts noted in the interviews; these concepts were formed from the grouping of similar attributes $[9,10]$. The attributes that were combined to form the concepts were given abbreviations such as P1.1, P1.2, P1.3, etc. For example, the concept of outdoor activity was composed of attributes such as 'fan dance', 'Tai Chi', 'sword dance' and 'jogging'. In order to achieve inter-rater reliability, a colleague independently coded $10 \%$ of the whole transcripts; and then, an English version of the report was written and recorded.

\section{Results}

From the analysis of the various concepts identified, one theme emerged: exercise among older adults who were attending senior activity centers (Figure1). The exercise theme from the interviews included three major concepts: outdoor activity, indoor activity, and other activity. All the older adults agree that their active life was greatly helpful in their daily life. The more exercise they performed, the more they liked and the more they would do which resulted that a group of older adults had two rounds of exercise (outdoor and indoor regular activity) in the morning in a senior activity center. Two groups of older adults living in Taipa and Macao peninsula, would have "Tai Chi" exercise in the outdoor garden. Then they would have their breakfast and returned to senior activity center for physical exercise again. The older adults were full of energy even they did not have any regular exercise attending in the senior activity center. They would go to the grocery early in the border of Macao and China where they could have less expensive food. They would buy lots of vegetables and meat two or three times a week. Some older adults would go to the grocery in the late evening as the food were cheaper for they were not so fresh. Some older adults not attending either indoor or outdoor activity, they had great responsibility. That was they needed to care their grandchildren. They had to prepare breakfast for grandchildren whom were primary children. Then they needed to take them to school in walk. In the afternoon, they had to fetch them back from school and prepare dinner for their adult children and grandchildren. 


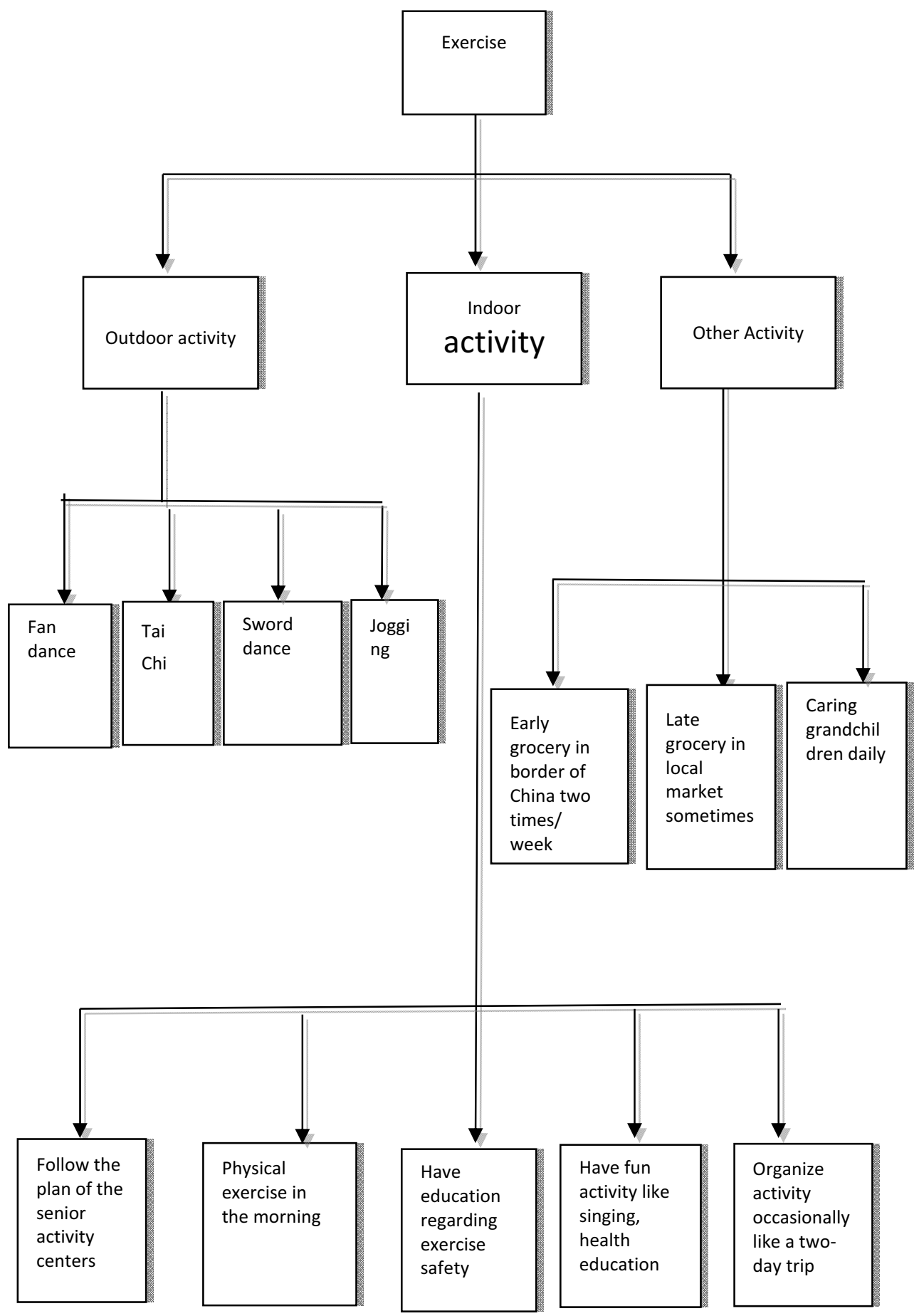

Fig. 1. Participation in healthy activity 


\subsection{Exercise}

\subsubsection{Outdoor activity}

Outdoor activity was the most important concept forming theme 1 (figure1). Older adults preferred to have outdoor activities early in the morning around seven o'clock. Adults from a senior activity center in Taipa and another senior activity center preferred to have regular exercise outdoor and indoor twice per day. The senior activity center started activity around nine in the morning. Since adults regularly had early wake up schedule. Therefore, they had the following comments:

'I have been doing exercise regularly after retirement, Before retirement, I occasionally performed exercise, I was quite lazy, because after the whole day working, I preferred watching TV lying in the sofa'. (P01-male/71)

'When I was young, I only performed exercise when I had time. Now I am a Tai Chi teacher, teaching some adults in a public garden... After I have breakfast with my wife and some Tai Chi friends, my wife and I will go back to center for exercise again. Then in the afternoon, every Tuesday and Thursday, I will learn Latin dance. Sometimes, we will go for competition... just going to mainland China for dance competition'. (P08-male/86)

'I did exercise twice, early morning in the outdoor, then after going back to center for second round..of course I will have breakfast, center opens around 9. My first round of exercise is around 7'. (P015 - female/84)

'I have fan dance as outdoor activity with some friends, we also practice sword dance. Mostly two times of both dances takes approximately 40 minutes, our center does not have regularly exercise activity provided'. (P28 - female/88)

\subsubsection{Indoor activity}

A senior activity center provided interesting activities in the afternoon such as singing, dancing, health knowledge session by professional specialist. The second center occasionally provided gathering and short trip such as a day or two-day trip to mainland China. The third and forth senior activity centers provided morning regular exercise program. One regular exercise activity was from Monday to Saturday while the other provided three times a week. The rest of the days, they may provide useful knowledge regarding prevention of musculoskeletal harm. They regularly provided healthy education such as how to prevent risk of falls, how to do exercise in a correct manner, and how to prevent harmfulness during physical exercise. The center's physical therapist would regularly provide proper information or correct their myth of how to do healthy and not risky exercise and activity.

'I will do more exercise after my husband's retirement, I will come to the center only in the afternoon, I need to prepare breakfast and dinner for my husband, he is working even he is 70 , he works in a private small business firm, so no retirement consideration, unless your boss thinks you are no more helpful'. (P22 - female/67)

'I like this center because they teach us what kind of exercise we can do, we know we are not young any more, risk of fall happens easily, we cannot bend like this... (stand up showing she cannot bend her upper body to legs while standing up'. (P30 - female/80)

'I seldom do extra exercise now, it is tiring to get up to this center (located in a top hill), I mean the outdoor exercise, here this center's exercise activity is simpler and easier, the staff will adjust our physical ability to match the level...'. (P34 - female/90)

'I occasionally have a jogging in the morning if it is not raining, however, I prefer going to swimming, I go to a swimming pool in Taipa, many adults swim there in the morning, I 
take bus, it is easy...in the afternoon, we have fun activity like singing, health education and discussion, or planning for activity and trip'. (P42 - female/82)

\subsubsection{Other activity}

These adults, whose center, mostly provided a short term trip for them and sometimes evening activity. So they are happy because they may be out of Macao for fun and do not have to care their family members and grandchildren. They were not having "sendary life". Their daily activity was caring family members, adult children and grandchildren such as going to grocery, doing meal preparation and taking grandchildren to school.

'I accompany my wife for grocery in the morning, grocery in the border is a good activity. We bought lots, my son and his family members live with us...'. (P40 - male/78).

'I now go to grocery in the late evening, but go to border buying meat and fish, there (border) is much cheaper and fresh'. (P48 - female/85).

'I have no time for activity, I have to bring my grandchildren (6 and 8 years old to school and back home in the afternoon. Their grandpapa will bring them back after school, sometimes. I have to climb up stairs to the $5^{\text {th }}$ floor'. (P50 - female/68)

'I have to prepare three meals a day, how I can have time for activity. My grandchild lives with us'. (P54 - female/72)

\subsection{Discussion}

The interviews conducted with 56 older adults provided a great number of insights into factors that may be contributed to their daily activity which might have effect to their choice of regular exercise. In addition, the senior activity center the older adults attended due to closer to their living area. However, the activity provided for the older adults also affected the favour of the older adults to healthy exercise. Continuously moving and having physiologic function is very important to the older adults [11]. The well-being of physical function significantly has great impact on older adults' quality of life. Physical functions mostly have the domains of daily activities such as self-care, bathing, dressing or walking and instrumental activities of daily living such as cooking, grocery and laundry [12]. Basically, walking as one wants or the higher level of doing exercise as one likes would be the top level of an older adult's independence and feeling of psychosocial wellness too. For many older adults, risk of falls and consequence of fractures play a very essential part to their intention of healthy physical activity.

As mentioned by participants, they would like to "move as they prefer". The activity center is a good venue providing an opportunity, environment and atmosphere for older adults to share and enjoy, and get useful healthy knowledge. Since the older adults have more time for learning and participating in their interested plans in comparison to their nonretirement status. A 78-year-old male participant stated: "I am more concerned about my health than before, I spend two hours doing exercise in the center, I use their activity instruments in the afternoon as there are not many people using. I also spend 30 minutes to massage my head, I can see things very well, no cataract in my eyes". The physiotherapist in the senior center always reminds and corrects the older adults' improper exercise or myth during activity. There the importance role of the senior activity did much support to older adults' physical and physiological development. It is because after learning the professional knowledge, they gained happiness, comfort and no harm after physical activity. They have more topics shared with their peer groups. They can sleep well after physical activity [13]. An 86-year-old female participant mentioned that if there was rain, she could not go and only practice "indoor activity". She felt uncomfortable as she mentioned. Other older adults 
felt they used to have exercise and felt good every day. So if there was rain for a few days, they stated that their bodies "did not feel easy and body is "itchy".

However, even some older adults have to take care of their grandchildren. That is one of the Chinese traditional cultures, a very good and tied union family support [14]. However, they do not lack walking and running up and down. Actually, walking and climbing upstairs and go to grocery or shopping is a good practicing of exercise [15]. It is interested to note that a senior activity center has the history of a couple of fall incidents of older adults. Maybe this is coincident to this study. The center rarely provides musculoskeletal function knowledge to the older adults. No matter what, the more we know, the more we benefit. The musculoskeletal function is also important during physical activity. Having proper exercise performance helps improves the flexibility of the strength of the muscle, tender, blood circulation and body system [16]. Older adults even have good benefit for mobility balance as well as muscle tone up. All the participants whom having regular exercise, mentioned that they felt happier, relaxed, energetic and not much body pain such as back or knee or foot [17]. They learned not to over use their joints and know how to protect them as they learned from center teaching class. However, those older adults caring grandchildren and performing much of household chores complaint of back pain and knee pain [14]. They need to carry heavy food or grandchildren whom were very small kids. Plus they lacked proper working body behaviour.

The 13-male participants all lived with their spouse, however, their spouses were typically no longer young themselves. They may have chronic illness or are very frail either. Therefore, there is a push for these male participants urged to be healthy and in return assist their spouses to care the family matters. From the qualitative approach, the results show that regular and proper exercise not only give the benefit to the older adult himself or herself, but also to the family members. In addition, with more formal physical activity knowledge provided by professionalism, the older adults enjoy more in physical and psychosocial development.

\subsection{Conclusion}

Healthy older adults usually have regular activities in the morning either outdoor or indoor or both after each other. Because of different family background, some older adults did not go for physical activities, they went for "other activity" for grocery, either in Macao border in the morning or late afternoon in local market as the vegetables were less expensive. The senior activity center also has great impact on them. If the center is more active in providing physical activity and educate the older adults how to involve suitable and healthy exercise, the older adults would be more enjoyable. They would not easily put them in risk of musculoskeletal twist and harm. As they mentioned they were more bravely to do exercise and were not afraid of hurting themselves. Therefore, providing regular health education regarding the exercises for older adults involved as mentioned above is very helpful in maintaining and improving gait, and balance. In addition, safety of exercise is

very important. Repetition of education on the function of musculoskeletal, such as how to protect the joints and bones, and musculoskeletal-related physical excise is vey essential. The old way of thinking needs to be replaced by new scientific thinking slowly.

\section{Acknowledgements}

The author would like to thank all the older adults for kindly participation in the interviews as well as the director of senior activity centers providing a comfortable and quiet area for interview. The author would also like to thank Macao Polytechnic Institute for funding this study ((RP/ESS -03/2014). 


\section{References}

1. M.E.Z. Estes, Health Assessment \& Physical Examination. Publisher: Cengage Learning (2014)

2. WHO Physcial Activity and Older Adults. Retrieved on 2017/06/10 website: http://www.who.int/dietphysicalactivity/factsheet_olderadults/en/ (2016)

3. UK Chief Medical Officers' Guidelines: Physcial activity benefits adults and older adults. Stay Active, Stay Active (2011)

4. R.J. Shephard, T.J. Verde, S.G. Thomas, P. Shek, Physcial activity and the immune system Can J. Sport Sci. (1991)

5. inMotoin, The Role of Physical Activity in Diabetes Prevention and Control, 17, 2, March/April (2007)

6. Centers for Disease Control and Prevention, Be Active! Why is it important for people with diabetes to be physically active?

7. Statistics \& Census Service, Demographic statisitics: Government of Macao special administrative region (2016)

8. S.U.C. Leong, L.B. Clutter M.W. Ho, Empowering Non-family Caregivers of Older Adults in Macao Chinese Society: Combining Theory Knowledge Lesson and Basic Care Skill Laboratory

9. J. Corbin, A. Strauss, Basics of Qualitative Research (2nd ed). Los Angeles, CA: Sage Publication (2008)

10. E.V. Cruz, G. Higginbottom, The use of focused ethnography in nursing research. Nurse Researcher, 20, 4, 36-43 (2013)

11. C.A. Miller, Nursing for wellness in older adults. Publisher : Wolters Kluwer (2015)

12. J.T. Stone, J.F. Wyman \& S.A. Salisbury, Clinical Gerontological Nursing: a guide to advanced practice. Publication: W.B. Saunders (1999)

13. M. Mendelson, et al., Low Physical Activity Is a Determinant for Elevated Blood Pressure in High Cardiovascular Risk Obstructive Sleep Apnea, Resp Care, 59, 8, 1218-1227 (2013)

14. J. Sun Chinese Older Adults Taking Care of Grandchildren: Practices and Policies for Productive Aging, Ageing Int ,38, 58-70 (2013)

15. Y.H. Chang, R.C.Y. Chen, M.L. Wahlqvist \& M.S. Lee, Frequent Shopping by Men and Women Increases Survival in the Older Taiwanese population, J Epidemiol Community Health, 66, e20 (2012)

16. M.R. Franco, A. Tong, K. Howard, C. Sherrington, P.H. Ferreira, R.Z. Pinto \& M.L. Ferreira, Older People's Perspective on Participation in Physical Activity: a Systematic Review and Thematic Synthesis of Qualitative Literature, Br J Sports Med, 49, 1268$1276(2015)$

17. E. Sitthipornvorakul, P. Janwantanakul, N. Purepong, P. Pensri, A.J. Beek, The Association between Physical Activity and Neck and Low Back Pain: a Systematic Review, Eur Spine J 20, 677-689 (2011) 